\title{
Acute Effect of Morning and Afternoon Aerobic Exercise on Appetite of Overweight Women
}

\author{
Zahra Alizadeh $^{1,2} ;$ Masoumeh Mostafaee $^{1,2} ;$ Reza Mazaheri $^{1,2,} ;$ Shima Younespour $^{3}$ \\ ${ }_{2}^{1}$ Sports Medicine Research Center, Tehran University of Medical Sciences, Tehran, IR Iran \\ ${ }^{2}$ Department of Sports and Exercise Medicine, School of Medicine, Tehran University of Medical Sciences, Tehran, IR Iran \\ ${ }^{3}$ Department of Epidemiology and Biostatistics, Tehran University of Medical Sciences, Tehran, IR Iran \\ ${ }^{*}$ Corresponding author: Reza Mazaheri, Department of Sports and Exercise Medicine, School of Medicine, Tehran University of Medical Sciences, Tehran, IR Iran. Tel: +98-2188630227, \\ Fax:+98-2188003539, E-mail: mazaheri_md@tums.ac.ir \\ Received: September 30, 2014; Revised: November 2, 2014; Accepted: November 15, 2014
}

\begin{abstract}
Background: The best time of exercise along the day for weight management in overweight and obese patients is not determined. The time of exercise may influence its effect on appetite and food intake.

Objectives: The aim of this study was to compare the effects of two different times of exercise during the day on appetite, energy intake, and the rating of perceived exertion (RPE) on overweight women.

Patients and Methods: Fifty overweight female subjects were recruited in this interventional study. Two sessions of exercise were performed in the morning and afternoon with the target heart rate corresponding to the ventilatory threshold (VT). The appetite was evaluated with visual analogue scale, the energy intake was measured with 24 hours food record and the RPE was determined by visual Borg scale; these variables were compared between the two sessions.

Results: The behavior of appetite in relation to hunger, satiety, fullness, prospective food consumption, tendency to salty, savoury, sweet and fatty foods, did not change significantly after both exercise sessions $(P>0.05)$. Except for the satiety, no significant difference was found among changes in the appetite scores between the two exercise sessions. The median change in the satiety score of the morning exercise was significantly higher than that of the afternoon exercise $(5.5(-8.5,22.5)$ vs. $-1(-8,4.5)$ respectively, $\mathrm{P}=0.01)$. The median RPE value did not differ significantly between the morning and afternoon sessions $(13(12,14)$ vs. $13(12,13)$ respectively, $\mathrm{P}=0.46)$. There was no significant association between the time of exercise and the estimates of the carbohydrate $(P=0.41)$, fat $(P=0.23)$, protein $(P=0.13)$, and calorie intake $(\mathrm{P}=0.18)$.

Conclusions: One session of moderate intensity exercise disregarding the time of exercise did not affect appetite significantly. However, morning exercise may cause greater levels of satiety in comparison with afternoon exercise. Moderate intensity aerobic exercise did not alter the patterns of energy intake 24 hours following morning or afternoon exercise.
\end{abstract}

Keywords: Appetite; Obesity; Physical Activity

\section{Background}

Nowadays, obesity and overweightness are said to be among the most significant health problems in developing and developed countries (1). Generally, more than 400 million adults are classified as obese, a number that is estimated to double by 2015 (1). It seems behavioral and environmental factors like today's modern sedentary lifestyle together with high calorie intake can be among the major causes of such an outbreak of overweightness and obesity (2). Obesity and overweightness are defined based on Body Mass Index (BMI), which is calculated by dividing the body weight (kilogram) by the squared body height (meter ${ }^{2}$ ). In case the scale falls between $25 \leq$ and $<30$ or at the 85 th to 94 th percentile for gender and age, one is considered to be overweight and if it surpasses 30 or 95 percentile, it is defined as obesity $(3,4)$. Today's obesity treatment techniques mainly include proper diet, increase in the amount of physical activity, exercise, behavioral therapy, medicinal therapy, surgical operations and alternative medicine like acupuncture $(5,6)$. Among these, the role of physical activity and exercise are especially highlighted, owing to their lasting and independent effects on health $(5,6)$.

There is a variety of studies on acute effects of exercise on appetite and energy intake, with some controversies (7-11). Overall, the controversies show that only $19 \%$ of the studies reported an increase in the amount of energy intake after exercise, while 65\% did not yield any significant relation (10). In fact, individuals with normal weight as well as obese ones may respond differently to exercise and the intensity of exercise would produce incompatible results. It has been demonstrated that high intensity exercise causes a greater negative balance of energy com- 
pared to low intensity one (12). Intense exercise reduces hunger in short-time however; transient control of hunger caused by high intensity exercise may not happen by moderate intensity $(13,14)$. There is also other evidence as to the influence of regular daily exercise on appetite due to energy imbalance $(15,16)$. Researchers in one study found that an acute exercise event could cause an instant fullness but repeating it for 12 weeks would increase hunger during the day (17).

The time of exercise may influence its effect on appetite too, but there are only a few studies carried out in this regard. As an instance, in one study comparison between morning and afternoon exercise showed an increase in the level of appetite, yet there were no significant differences in appetite changes between the morning and afternoon groups; however, regardless of time, a shortterm improvement was observed in negative balance of energy (energy expenditure as opposed to energy intake) (18). RPE during exercise may be different in obese people, but there are only a few studies conducted in this area. In one study, morning exercise and evening exercise were compared, in which there was a higher rate of perceived exertion in the morning group (18).

\section{Objectives}

Concerning the limited volume of studies in this field and incompatible results and controversies in papers, the aim of this investigation is to compare the effects of one session of morning aerobic exercise with afternoon aerobic exercise (ME vs. AE) on appetite, energy intake, and the RPE in overweight women.

\section{Patients and Methods}

Fifty 20-45 years old overweight women were recruited in this interventional study. All subjects underwent two aerobic exercise sessions (morning and afternoon) to examine the effect of time of exercise on appetite, the amount of food consumed in the next 24 hours and the RPE. The order of the exercise sessions was randomly determined in each subject. Through advertising in public places, overweight women were recruited and selected via preliminary interviews and measurements in the multidisciplinary obesity clinic in the Imam Khomeini hospital.

The inclusion criteria were being female between 20 and 45 years of age; BMI equal to or greater than 25 and less than $30 \mathrm{~kg} / \mathrm{m}^{2}$; sedentary lifestyle (not participating in at least 30 minutes of moderate intensity exercise 3 days per week in the three months prior to the commencement of the study); not suffering from any known cardiovascular, pulmonary, or metabolic diseases; not taking any medication affecting heart rate, blood pressure or exercise capacity like beta-blockers or antihypertensive drugs; not experiencing musculoskeletal problems that would limit exercise capacity; and not being pregnant. In case a participant refused participation or had any indication of exercise test termination during the test, she would be excluded.

The evaluation of physical activity was done using interview and questions from participants. The food consumption was assessed with 24 hours food record that was self- administered but we completed the data with interview after the completion of the form. The appetite was assessed with self-administered 8-item visual analogue scale. The RPE was assessed with 6 - 20 score Borg scale. (The forms are attached in the appendix). After primary instructions including an explanation on exercise test protocol as well as ME and AE sessions, how to fill out the appetite visual analogue scale, and how to record the 24 hour diet record and the 6 - 20 score Borg scale for the RPE, the participants were asked to comply with the verbal and written informed consent. The second visit for exercise test with proper preparations was determined. They were asked to drink enough water, not to do exercise the day before the visit, and not eat three hours before the test.

In the second visit, the participants' heights were measured using a standard measuring tape. They were weighed on a medical scale with a precision of 100 grams, and the percentage of their body fat was calculated using Body Impedance Analyzer (AVIS33 body composition analyzer, Jawon Medical Co. Ltd, South Korea). On the day of the test, after calibrating the gas analysis device (Quark CPET, COSMED, Italy) the proper mask was worn by the participants and the exercise test was administered based on Bruce protocol. The test was continued until exhaustion. The VT was calculated according to the results of gas analysis and the participants' heart rate at the level of VT was recorded based on the percentage of maximal heart rate (HRVT (\%Max)).

Each participant, based on the table of random numbers, was included in the ME or AE groups in the second session. Afterwards, the next session was appointed for each group, which was supposed to be at least 72 hours away from the exercise test. In order to eliminate any physiological effects of high intensity exercise during the exercise test, the exercise sessions were done 72 hours after the test. The participants were asked to record their food intake in the 24 hours before the exercise sessions. In the third visit, the morning group returned from 8 to $10 \mathrm{AM}$ and the afternoon group from 2 to 4 PM, when they had to do aerobic exercise on treadmill for 30 minutes. The components of the exercise session were as follows: stage 1 , gradual increase in the speed of the treadmill (warming up for 5 minutes); stage 2, increasing the speed up to the VT that was calculated in the exercise test and running at the required speed for 20 minutes; stage 3, cooling down for 5 minutes. In the peak of the exercise session, the participants were asked about the RPE. They were also required to fill out the 0 - 100 millimeter appetite visual analogue scale, with its proved validity and reliability (19), to measure the level of hunger, fullness, satiety, prospective food consumption, tendency to salty, 
savoury, sweet, and fatty foods before the exercise and 15 minutes afterward.

The amount of drunk water after the exercise session and before completing the logbook was recorded and the participants were asked to record their food intake in the next 24 hours after the session. All the participants were participated in the second exercise session after 72 hours in the opposite time (morning participants would come in the afternoon and vice versa) and the program was repeated all over. The 24 hours food records were analyzed using the FPI II software (Food Processor II, Nutrition System ESHA Research, Salem, Oregon 1987) which could calculate the consumed calorie, carbohydrate, protein and fat intake based on percentage and grams. Finally, basic 24 hour records were compared with records after ME and AE. This research has been approved by the ethical committee in Tehran University of Medical Sciences.

\subsection{Statistical Analysis}

The statistical software SPSS 16.0.0 (SPSS Inc. Chicago, IL, USA) was used for data analyses. P values less than 0.05 were considered statistically significant. All tests were two-sided. To study the impact of time of exercise (ME and $\mathrm{AE}$ ) on the changes of appetite scores (post-trial appetite score minus pre-trial appetite score), we decided to conduct a study that would be analyzed using the Wilcoxon signed-rank test. A sample size of 50 achieves $80 \%$ power to detect a difference of $-10 \mathrm{~mm}$ between the null hypothesis mean (the mean difference between changes of appetite scores of ME and AE) of 0.0 and the alternative hypothesis mean of $10 \mathrm{~mm}$ with an estimated standard deviation of $25 \mathrm{~mm}$ and with a significance level (alpha) of 0.05 using a two-sided Wilcoxon test.

Categorical data were expressed as numbers (percentages). Continuous variables were reported as mean \pm SD or as medians with interquartile ranges (25th, 75 th percentiles). The normality assumption of the continuous variables was assessed using the Shapiro-Wilk W-test. Subjective feelings of appetite and desired scores before and after exercise sessions were compared by the nonparametric Wilcoxon signed-rank test. This test was also applied for the comparisons between changes in the appetite scores (post-trial appetite score minus pre-trial appetite score) of ME and AE trials.

In this study, each subject had three measurements for dietary intakes (at baseline and after ME and $\mathrm{AE}$ sessions). Using the Generalized Estimating Equation (GEE) (GEE) models, the correlation of multiple measurements within one subject is taken into account. In our GEE models, the time of exercise is considered as fixed factor and the estimates of the carbohydrate, fat, protein, and calorie intake (obtained by 24 hour food record) are considered as dependent variables. We used (GEE) models with an exchangeable correlation matrix to examine the effect of time of exercise (at baseline and after ME and AE sessions) on the estimate of the carbo- hydrate, fat, protein, and calories consumed by the participants.

\section{Results}

Fifty overweight female subjects were recruited in this study. Four of the participants did not participate in the exercise sessions due to lack of enough time. Finally, a total of forty-six subjects were analyzed. Baseline characteristics and anthropometric measurements of the participants are summarized in Table 1. Participants' average maximum heart rate during exercise test (mean \pm SD) was $178.35 \pm 9.27$ beats per minute; the percentage of maximal heart rate during exercise test was $97.64 \pm 4.41$; estimated mean heart rate during $\mathrm{ME}$ and $\mathrm{AE}$ sessions was $150.51 \pm 9.57$ beats per minute; the mean percentage heart rate during exercise sessions was $84.28 \pm 3.57$; and the mean speed during ME and AE sessions was $6.30 \pm$ $0.31 \mathrm{~km} /$ hour. The median RPE score did not differ significantly between the ME and AE trials $(13, \operatorname{IQR}(12,14)$ vs. 13 $\operatorname{IQR}(12,13), P=0.46)$. There was no significant difference in the median liquid consumption of the subjects after ME and AE sessions (median 225 cc, IQR $(180,360)$ cc for both exercise sessions, $\mathrm{P}=0.73$ ).

The behavior of appetite in relation to hunger, satiety, fullness, prospective food consumption and desire for sweet, salty, savoury and fatty foods did not change significantly by aerobic exercise in both ME and AE sessions (Table 2). Except for the satiety, no significant difference was found between changes in the appetite scores of the ME and AE sessions (Table 2). The median change in the satiety score (post-trial satiety score minus pre-trial satiety score) of the ME trial was significantly higher than that of the AE trial (median 5.5, IQR $(-8.5,22.5)$ vs. median $-1, \operatorname{IQR}(-8,4.5) ; \mathrm{P}=0.01$ in Figure 1). The estimates of the carbohydrate, fat, protein, and calorie intake (obtained by 24 hour food record) at baseline and after ME and AE sessions are summarized in Table 3. According to the results of GEE analyses, no significant association was found between these items and the time of exercise (Table 3).

Table 1. Baseline Characteristics and Anthropometric Measurements of the Participants a,b

\begin{tabular}{lc}
\hline Variables & Values \\
\hline Age, $\mathbf{y}$ & $33.37 \pm 6.08$ \\
\hline Height, $\mathbf{c m}$ & $161.51 \pm 4.52$ \\
\hline Weight, $\mathbf{k g}$ & $71.59 \pm 6.51$ \\
\hline BMI ${ }^{\mathrm{C}}, \mathbf{k g} / \mathbf{m}^{\mathbf{2}}$ & $27.40 \pm 1.52$ \\
\hline Body fat, \% & $35.57 \pm 2.12$ \\
\hline $\begin{array}{l}\text { a Data are expressed as mean } \pm \text { SD. } \\
\text { b Abbreviation: BMI, body mass index. } \\
\text { c Calculated as weight in kilograms divided by height in meters } \\
\text { squared. }\end{array}$
\end{tabular}


Alizadeh Z et al.

\begin{tabular}{|c|c|c|c|c|c|c|c|c|c|}
\hline \multirow[t]{2}{*}{ Variables } & \multicolumn{4}{|c|}{ ME Trial } & \multicolumn{4}{|c|}{ AE Trial } & \multirow[t]{2}{*}{ PValue } \\
\hline & Pre-Trial & Post-Trial & Change & PValue $^{\mathrm{d}}$ & Pre-Trial & Post-Trial & Change & PValue $^{\mathrm{e}}$ & \\
\hline Satiety & $74.5(37.25,92.5)$ & $85.5(48.5,100)$ & $5.5(-8.5,22.5)$ & 0.11 & $80(44.25,100)$ & $87(38.5,100)$ & $-1(-8,4.5)$ & 0.38 & 0.01 \\
\hline Hunger & $10.5(0,33.75)$ & $4(0,29.5)$ & $0(-8,6.5)$ & 0.76 & $6(0,33.25)$ & $7(0,26.5)$ & $0(-6.5,5)$ & 0.51 & 0.54 \\
\hline Fullness & $69(33.25,90)$ & $76.5(48.25,94.5)$ & $0(-5,22.3)$ & 0.18 & $79.5(43.25,98.25)$ & $78(49.5,98)$ & $0(-6.5,2.5)$ & 0.52 & 0.40 \\
\hline PFC & $29.5(8.75,58.25)$ & $19(1,48.25)$ & $0(-29.25,6.25)$ & 0.12 & $23(1,50)$ & $16(2,35)$ & $0(-10.5,3.75)$ & 0.14 & 0.19 \\
\hline Salty & $99(86.25,100)$ & $100(89,100)$ & $0(-0.25,2)$ & 0.50 & $99(84,100)$ & $99(87.5,100)$ & $0(-1,1.5)$ & 0.60 & 0.79 \\
\hline Sweet & $78(24,94.25)$ & $74.5(25.75,100)$ & $1(-10,15.5)$ & 0.41 & $57(29.5,99.25)$ & $80(27,100)$ & $0(-8.5,7.5)$ & 0.79 & 0.97 \\
\hline Savoury & $100(84.75,100)$ & $100(92.25,100)$ & $0(0,1.25)$ & 0.18 & $99(73.5,100)$ & $94(81.5,100)$ & $0(-0.5,2)$ & 0.55 & 0.60 \\
\hline Fatty & $100(97.5,100)$ & $100(98,100)$ & $0(0,1.25)$ & 0.10 & $100(96,100)$ & $100(92,100)$ & $0(0,0.5)$ & 0.41 & 0.44 \\
\hline $\begin{array}{l}\mathrm{a} \text { Abbreviat } \\
\mathrm{b} \text { The appet } \\
100 \text { mm line } \\
{ }^{c} \text { P value for } \\
d \text { P value fo } \\
e^{\text {e value for }}\end{array}$ & $\begin{array}{l}\text { ons: AE, Afternoon } \\
\text { ite and desire scor } \\
\text { The values are exp } \\
\text { comparison betwe } \\
\text { comparison betw } \\
\text { comparison betwe }\end{array}$ & $\begin{array}{l}\text { Exercise; ME, Mor } \\
\text { es for specific food } \\
\text { oressed as median } \\
\text { een changes in the } \\
\text { een pre- and post- } t \\
\text { een pre-and post- } t\end{array}$ & $\begin{array}{l}\text { ning Exercise; } P \\
\text { type were asse } \\
\text { (25th, } 75 \text { th per } \\
\text { appetite score } \\
\text { trial appetite sc } \\
\text { trial appetite sc }\end{array}$ & $\begin{array}{l}\text { PFC, Prospec } \\
\text { ssed using } \\
\text { centiles). } \\
\text { s (post-trial } \\
\text { cores in the }\end{array}$ & $\begin{array}{l}\text { ctive Food Consum } \\
\text { a self-administrate } \\
\text { lappetite score min } \\
\text { ME session. } \\
\text { AE session. }\end{array}$ & $\begin{array}{l}\text { tion. } \\
\text { questionnair } \\
\text { is pre-trial app }\end{array}$ & $\begin{array}{l}\text { the form of } \mathrm{v} \\
\text { te score) of } \mathrm{M}\end{array}$ & $\begin{array}{l}\text { yal analog } \\
\text { and AE sess }\end{array}$ & $\begin{array}{l}\text { e scales of } \\
\text { ons. }\end{array}$ \\
\hline
\end{tabular}

Table 3. Summary of 24 Hour Food Records at Baseline and After Morning and Afternoon Exercise Sessions a,b,c

\begin{tabular}{lccc}
\hline Variables & Baseline & After ME Session & After AE Session \\
\hline Total energy intake, kcal & $1949.67 \pm 507.13$ & $2079.17 \pm 576.53$ & $1920.40 \pm 443.42$ \\
Protein, gr & $65.99 \pm 22.99$ & $60.43 \pm 18.08$ & $59.07 \pm 17.45$ \\
Energy from protein, \% & $13.71 \pm 4.81$ & $12.00 \pm 4.16$ & $12.33 \pm 2.58$ \\
Carbohydrate, gr & $241.89 \pm 84.11$ & $260.35 \pm 92.34$ & $245.66 \pm 72.04$ \\
Energy from carbohydrate, \% & $48.75 \pm 7.75$ & $49.78 \pm 6.80$ & $50.41 \pm 7.57$ \\
Fat, gr & $82.64 \pm 28.32$ & $90.30 \pm 32.34$ & 0.13 \\
Energy from fat, \% & $37.47 \pm 8.29$ & $38.19 \pm 7.38$ & 0.52 \\
\hline
\end{tabular}

$\mathrm{a}$ The values are expressed as mean $\pm \mathrm{SD}$.

b Abbreviations: AE, Afternoon Exercise; ME, Morning Exercise.

C P values obtained by GEE models to assess the effect of time of exercise (at baseline and after morning and AE sessions) on the estimate of the carbohydrate, fat, protein and calorie intakes.

Figure 1. Change in the Satiety Score (Post-Trial Satiety Score Minus PreTrial Satiety Score) of the Morning and Afternoon Eexercise Sessions.

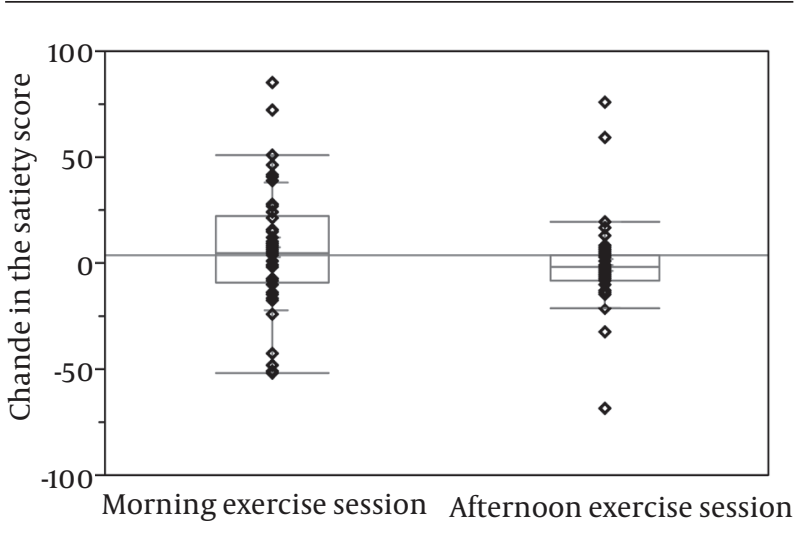

Time of exercise

Middle point: median; Box: interquartile range (25 - 75 percentiles); Whisker: range (excluding outliers).

\section{Discussion}

The present investigation is an experimental study observing 50 overweight women with sedentary lifestyle. Except in satiety, there was no significant statistical difference in appetite scores after ME and AE sessions. The score of satiety changed more in the morning session compared to that of the afternoon. Imbeault et al. (12) reported from their study on 11 young males that one session of exercise did not have an effect on the feelings of hunger, fullness, and energy intake. Hubert et al. (8) also showed that exercise was ineffective on appetite. On the other hand, King and colleagues' study on 24 young men showed that cycling and running caused a temporary decrease in hunger (20). Broom et al. (21) embarked on comparing the effects of aerobic exercise with resistance training on 11 healthy men and concluded that both types of exercise caused a decrease in hunger sensation and ghrelin. There is limited bulk of study comparing satiety scores of morning with those of the evening or afternoon and most of the researches have measured the effects of 
exercise on appetite independent of time (18). Maraki et al. (18) demonstrated that one session of exercise done in the morning or evening changed appetite patterns significantly (increase in hunger, decrease in satiety and feelings of fullness). They expressed that after one session of exercise the level of appetite would increase, which is incompatible with the findings of our study. The incompatibility can be justified as the fact that in our study appetite was evaluated immediately after exercise, while in the study carried out by Maraki et al. (18) participants did an aerobic exercise for 20 minutes, muscle conditioning exercise for 20 minutes, and cooling down for 10 minutes. Then, the feelings of appetite were evaluated after cool down (45 minutes after the end of the aerobic exercise). They also employed two types of exercise including aerobic phase and a muscle conditioning phase while in our study only one type was used. In 2010, Martins and colleagues found in their study on 22 overweight and obese individuals after 12 weeks of exercise that exercise could control appetite (22). In general, the results of studies on the effects of one exercise session on appetite are various and a matter of controversy; the reason however, can be due to different exercise programs including variant levels of exercise intensity. Mostly, it seems that the transient control of appetite, which comes by intense exercise, simply does not occur as a result of moderate intensity (23). After all, considering the present study, it appears that moderate intensity exercise cannot affect appetite in the morning and afternoon acutely, yet individuals may feel more satiety after ME compared to that of the afternoon. Although the decrease in satiety score did not led to a decrease in 24 hours post exercise food consumption, but this change may be an important item during the long term, since the lifestyle changes in overweight and obese individuals need time. The further researches need to evaluate the long-term effect of decreased satiety sensation after morning exercise on weight reduction.

In our study, calculations of carbohydrate, fat, protein, and the consumed calorie (based on the 24 hour food records) at the baseline and after ME and AE did not yield any significant differences. Other studies also showed that energy intake following exercise did not change (18, 24). For instance, Maraki et al. (18) found that exercise did not change energy intake following a training session. In 2008, Dodd and colleagues reported that the level of food intake after exercise did not increase in normal weight and overweight girls (24). King et al. (20) studied 24 young males and uttered that cycling and running did not influence the total amount of food intake (calorie per day) significantly. Yet, the two types of exercises altered their lunching habits so that their level of fatty, low-carbohydrate food intake increased significantly. Woo and colleagues analyzed energy intake following an increase in the level of physical activity; the study was carried out on six overweight women who used treadmill over a 19 day exercise program. The results showed that the individuals experienced a negative balance of energy; in oth- er words, while exercise caused an increase in the level of energy expenditure, the compensatory increase in energy intake did not occur (25). This, in fact, was compatible with our study. Bellissimo et al. (26) analyzed the effects of short-term physical activity on appetite and energy intake in 14 male teenagers with normal weight and found out that appetite would increase following walking on treadmill while food intake after exercise decreased compared to the control group. In general, many studies have shown that exercise does not increase the level of energy intake, even with high intensity exercise and thus it can make a negative balance of energy in short time (23). Our study, however, was compatible with the aforementioned researches and demonstrated that moderate intensity exercise done either in the morning or in the afternoon did not raise the level of energy intake 24 hours after exercise. Further, it showed that the pattern of food intake did not alter in terms of macronutrient intake levels following morning or evening moderate intensity aerobic exercise.

As stated before, the present study did not observe any significant statistical difference in the mean of the perceived exertion ratings among the participants in ME and AE. Few studies have examined the effect of time of day on the RPE of exercise $(18,27)$. Our study, however, is in line with that conducted by Hill and colleagues in which no difference was observed in the RPE of morning and afternoon exercise at intensities below the VT (27). In 2005, Maraki and colleagues measured the RPE in warm up, aerobic exercise, and resistance training among 12 women with normal BMI who did not follow regular exercise programs and found out that the morning group perceived higher rates of exertion compared to the afternoon counterparts in warm up and resistance training but not in the aerobic phase. They discussed that as a result of early morning exercises, the participants were not up and about; that is why they scored higher RPE. They also considered lower body temperature and level of flexibility in the morning as other probable causes of the difference (18). Our study, on the other hand, did the warm-up stage on treadmill and the RPE was evaluated on the peak of aerobic exercise, thus there was compatibility. After all, there was no significant difference in the RPE among the overweight women at moderate intensity morning aerobic exercise session compared with those of the afternoon.

In the present study the mood changes and the influence of menstruation cycle on the participant's appetite were not assessed. It should be noted that in the study there was no measuring of the blood levels of hormones including those involved in appetite control like ghrelin. However, analysing respiratory gases and identifying every individual's ventilatory threshold during exercise test were the strength of the study, so every participant's exercise program was prescribed based on this item and the moderate intensity supervised aerobic exercise on treadmill was done along the study. 
The present study demonstrated that among a group of healthy but overweight women with sedentary lifestyle, one session of moderate intensity exercise disregarding the time of the exercise (morning or afternoon) did not affect appetite (the feelings of hunger, fullness, satiety, prospective food consumption, tendency to salty, savoury, sweet, and fatty foods) significantly. However, ME may cause greater levels of satiety in comparison with AE. Further, moderate intensity aerobic exercise on treadmill did not alter the patterns of energy intake and the consumption of macronutrients (carbohydrates, proteins, and fat) 24 hours following exercise. The study also did not show any significant difference in terms of the RPE in the ME and $\mathrm{AE}$.

\section{Authors' Contributions}

Zahra Alizadeh: study concept and design, administrative, technical, and material support, study supervision and interpretation of data. Reza Mazaheri: critical revision of the manuscript for important intellectual content. Masoumeh Mostafaee: drafting of the manuscript. Shima Younespour: analysis and interpretation of data and Statistical analysis.

\section{Funding/Support}

The funding for the project was provided by Tehran University of Medical Sciences, Faculty of Medicine, Department of Sports and Exercise Medicine (grant number 18184-53-02-91). Department of sports and exercise medicine in Imam Khomeini Hospital provided practical support for the survey.

\section{References}

1. Shea J, Diamandis EP, Sharma AM, Despres JP, Ezzat S, Greenway F. The obesity epidemic. Clin Chem. 2012;58(6):968-73.

2. Charansonney OL, Despres JP. Disease prevention--should we target obesity or sedentary lifestyle? Nat Rev Cardiol. 2010;7(8):468-72.

3. Barlow SE, Expert C. Expert committee recommendations regarding the prevention, assessment, and treatment of child and adolescent overweight and obesity: summary report. Pediatrics. 2007;120 Suppl 4:S164-92.

4. World Health Organization. Global Database on Body Mass Index. Geneva: WHO;2006.

5. Bouchard C, Depres JP, Tremblay A. Exercise and obesity. Obes Res. 1993;1(2):133-47.

6. Shaw K, Gennat H, O'Rourke P, Del Mar C. Exercise for overweight or obesity. Cochrane Database Syst Rev. 2006;(4):CD003817.

7. King NA, Tremblay A, Blundell JE. Effects of exercise on appetite control: implications for energy balance. Med Sci Sports Exerc. 1997;29(8):1076-89.

8. Hubert P, King NA, Blundell JE. Uncoupling the effects of energy expenditure and energy intake: appetite response to short-term energy deficit induced by meal omission and physical activity. Appetite.1998;31(1):9-19.
9. King NA. What processes are involved in the appetite response to moderate increases in exercise-induced energy expenditure? Proc Nutr Soc. 1999;58(1):107-13.

10. Blundell JE, King NA. Physical activity and regulation of food intake: current evidence. Med Sci Sports Exerc. 1999;31(11 Suppl):S573-83.

11. King NA, Caudwell P, Hopkins M, Byrne NM, Colley R, Hills AP, et al. Metabolic and behavioral compensatory responses to exercise interventions: barriers to weight loss. Obesity (Silver Spring). 2007;15(6):1373-83.

12. Imbeault P, Saint-Pierre S, Almeras N, Tremblay A. Acute effects of exercise on energy intake and feeding behaviour. Br J Nutr. 1997;77(4):511-21.

13. King NA, Burley VJ, Blundell JE. Exercise-induced suppression of appetite: effects on food intake and implications for energy balance. Eur J Clin Nutr. 1994;48(10):715-24.

14. Stensel D. Exercise, appetite and appetite-regulating hormones: implications for food intake and weight control. Ann Nutr Metab. 2010;57 Suppl 2:36-42.

15. Stubbs RJ, Hughes DA, Johnstone AM, Whybrow S, Horgan GW, King N, et al. Rate and extent of compensatory changes in energy intake and expenditure in response to altered exercise and diet composition in humans. Am J Physiol Regul Integr Comp Physiol. 2004;286(2):R350-8.

16. Whybrow S, Hughes DA, Ritz P, Johnstone AM, Horgan GW, King $\mathrm{N}$, et al. The effect of an incremental increase in exercise on appetite, eating behaviour and energy balance in lean men and women feeding ad libitum. Br J Nutr. 2008;100(5):1109-15.

17. King NA, Caudwell PP, Hopkins M, Stubbs JR, Naslund E, Blundell JE. Dual-process action of exercise on appetite control: increase in orexigenic drive but improvement in meal-induced satiety. Am JClin Nutr. 2009;90(4):921-7.

18. Maraki M, Tsofliou F, Pitsiladis YP, Malkova D, Mutrie N, Hig gins S. Acute effects of a single exercise class on appetite, energy intake and mood. Is there a time of day effect? Appetite. 2005;45(3):272-8.

19. Flint A, Raben A, Blundell JE, Astrup A. Reproducibility, power and validity of visual analogue scales in assessment of appetite sensations in single test meal studies. Int J Obes Relat Metab Disord. 2000;24(1):38-48.

20. King NA, Blundell JE. High-fat foods overcome the energy expenditure induced by high-intensity cycling or running. Eur J Clin Nutr. 1995;49(2):114-23.

21. Broom DR, Batterham RL, King JA, Stensel DJ. Influence of resistance and aerobic exercise on hunger, circulating levels of acylated ghrelin, and peptide YY in healthy males. Am J Physiol Regul Integr Comp Physiol. 2009;296(1):R29-35.

22. Martins C, Kulseng B, King NA, Holst JJ, Blundell JE. The effects of exercise-induced weight loss on appetite-related peptides and motivation to eat. JClin Endocrinol Metab. 2010;95(4):1609-16.

23. King JA, Miyashita M, Wasse LK, Stensel DJ. Influence of prolonged treadmill running on appetite, energy intake and circulating concentrations of acylated ghrelin. Appetite. 2010;54(3):492-8.

24. Dodd CJ, Welsman JR, Armstrong N. Energy intake and appetite following exercise in lean and overweight girls. Appetite 2008;51(3):482-8.

25. Woo R, Garrow JS, Pi-Sunyer FX. Effect of exercise on spontaneous calorie intake in obesity. Am J Clin Nutr. 1982;36(3):470-7.

26. Bellissimo N, Thomas SG, Goode RC, Anderson GH. Effect of shortduration physical activity and ventilation threshold on subjective appetite and short-term energy intake in boys. Appetite 2007;49(3):644-51.

27. Hill DW, Cureton KJ, Collins MA. Effect of time of day on perceived exertion at work rates above and below the ventilatory threshold. Res Q Exerc Sport. 1989;60(2):127-33. 